\title{
Th1/Th2 cytokine imbalance in meningioma, anaplastic astrocytoma and glioblastoma multiforme patients
}

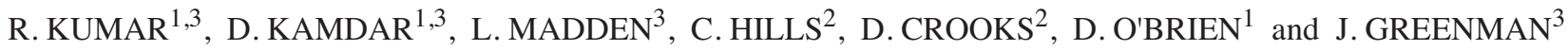 \\ Departments of ${ }^{1}$ Neurosurgery and ${ }^{2}$ Neuropathology, Hull and East Yorkshire Hospitals; \\ ${ }^{3}$ Department of Surgery, University of Hull, UK
}

Received February 24, 2006; Accepted March 31, 2006

\begin{abstract}
The balance between Th1 and Th2 cytokines is thought to be an important factor in terms of tumour prognosis. Serum samples from 61 newly diagnosed patients with brain tumours and 50 age- and sex-matched non-tumour controls were analysed by ELISA for circulating levels of interleukin-12 (IL-12p70 and p40) and interleukin-10 (IL-10); pivotal Th1 and Th2 cytokines, respectively. Patients were divided into various groups depending on their histological diagnosis: meningioma $(n=11)$, anaplastic astrocytoma $(n=4)$ and glioblastoma multiforme (GBM; $n=46)$. Significant reduction in serum IL-12 was seen in all groups as compared with the controls: meningioma, $\mathrm{p}=0.03$; anaplastic astrocytoma, $\mathrm{p}<0.001$; and GBM, $\mathrm{p}<0.001$. Conversely, serum IL-10 was significantly increased in anaplastic astrocytoma, $\mathrm{p}=0.02$, and GBM, $p=0.03$. The changes in the serum cytokines were not caused by the effects of steroids, as sequential analysis of patients pre- and post-steroid treatment commencement showed no difference. This study shows that patients with advanced primary intracranial malignancies have decreased circulating IL-12 and increased circulating IL-10, demonstrating that brain tumours have a major systemic effect on the immune system.
\end{abstract}

\section{Introduction}

Tumours of the brain account for $1.6 \%$ of cancers in the United Kingdom, being the twelfth most common type of cancer in men and the fifteenth in women. Survival figures have not changed significantly over the past 20 years despite advances in conventional therapy, (surgery, chemotherapy and radiotherapy), with $25 \%$ survival at 2 years after diagnosis and only $20 \%$ at 5 years $(1,2)$.

Correspondence to: Dr J. Greenman, Department of Surgery, Clinical Biosciences Institute, Wolfson Building, University of Hull, Cottingham Road, Hull HU6 7RX, UK

E-mail: j.greenman@hull.ac.uk

Key words: Th1/Th2 cytokine, meningioma, anaplastic astrocytoma, glioblastoma multiforme
The Th1/Th 2 model is a well-established way of understanding the various cytokines that are secreted by the different $\mathrm{CD}^{+} \mathrm{T}$ helper lymphocyte subsets. The CD4+ $4^{+} \mathrm{Th}$ helper population is generally pro-inflammatory and the cytokine profile includes interferon-gamma (IFN- $\gamma$ ), interleukin-2 (IL-2), IL-12, IL-15, lymphotoxin and tumour necrosis factoralpha $(\mathrm{TNF}-\alpha)$. In collaboration, these cytokines stimulate a cell-mediated response and, especially in vitro, have been shown to exert a potent anti-tumour effect. In contrast, $\mathrm{CD}^{+}{ }^{+} \mathrm{Th} 2$ cells produce cytokines such as IL-4, IL-10, IL-13 and stimulate the humoral immune response. The Th1 and Th2 cytokines act in an antagonistic fashion; with a balancepoint that varies between the physiological and pathological state (3).

IL-12 is a $70-\mathrm{kDa}$ disulphide-linked heterodimer, composed of two N-glycosylated polypeptide chains of approximately $40 \mathrm{kDa}(\mathrm{p} 40)$ and $35 \mathrm{kDa}$ (p35) linked by a single disulfide bond, which is principally produced by monocytes and macrophages (4). IL-12 induces IFN- $\gamma$ production, stimulates proliferation and enhances the cytolytic activity of natural killer (NK) and T-cells (5). IL-12 acts synergistically with IL-18 to induce IFN- $\gamma$ production in antigen presenting cells $(6,7)$. In addition to positively promoting cell-mediated immunity, it negatively regulates Th1 cell differentiation (8).

IL-12 has also been shown to be effective in preventing and inhibiting the growth of primary tumours induced by chemical carcinogens in in vitro models $(9,10)$. Furthermore, it has been shown to be effective in preventing and inhibiting the growth of primary tumours arising spontaneously in transgenic mice expressing rat HER-2/neu oncogene in the mammary gland (11). In addition to the in vitro and animal models, there is evidence for IL-12 causing an anti-tumour response in humans; however, toxicity is a problem $(12,13)$.

IL-10 is a 17 - to $21-\mathrm{kDa}$ cytokine principally produced by activated lymphocytes as well as some cancer cell types (14-16). IL-10 was originally coined CSIF (cytokine synthesis inhibitory factor) because of its general suppressive role; more specifically, its ability to block the synthesis of interferon-gamma (IFN- $\gamma$ ), IL-1 $\alpha$, IL-1ß and IL-12, amongst other cytokines. In addition to cytokine inhibition, IL-10 has also been shown to reduce the antigen presentation capacity of monocytes by down-modulating MHC class II expression, this in turn hinders antigen-stimulated proliferation of specific T-cells. The antagonistic role of IL-12 and IL-10 has been demonstrated in a variety of tumour types $(3,17,18)$. 
Table I. Clinicopathological features of patients and control group in Th1/Th2 study.

\begin{tabular}{lccccc}
\hline $\begin{array}{l}\text { Histopathology } \\
\text { (WHO grade) }\end{array}$ & $\begin{array}{c}\text { Median age } \\
\text { (years) }\end{array}$ & $\begin{array}{c}\text { Standard } \\
\text { deviation }\end{array}$ & $\begin{array}{c}\text { Interquartile } \\
\text { range }\end{array}$ & M:F ratio & $\begin{array}{c}\text { No. of } \\
\text { patients }\end{array}$ \\
\hline Meningioma (benign) & 39 & 15.5 & $33-76$ & $4: 7$ & 11 \\
Anaplastic astrocytoma (III) & 71 & 7.2 & $57-72$ & $3: 1$ & 4 \\
Glioblastoma multiforme (IV) & 60 & 10.8 & $39-75.7$ & $26: 20$ & 46 \\
\hline
\end{tabular}

Controls: median age, 59 years; standard deviation, 12.3; interquartile range, 37-81; male:female, 29:21. ND, not done.

The purpose of this study was to investigate the Th1/Th2 balance in patients with newly diagnosed brain tumours (meningioma, anaplastic astrocytoma and GBM), studying the levels of pivotal cytokines IL-12 (Th1) and IL-10 (Th2). These cytokines were selected because they play pivotal roles in determining the nature of the immune response. The aim is to determine if the levels of these cytokines or the relative balance changes in comparison with healthy controls and between tumour stage.

\section{Materials and methods}

Patients details. Serum samples were obtained from 61 patients with brain tumours, and these were compared with a cohort of 50 non-tumour controls of similar age and male: female ratio. The control group were patients electively admitted for surgical treatment of degenerative spines. They did not have any pre-existing tumours, nor had received any radiotherapy or chemotherapy within the past year. Ethical approval was obtained from Hull and East Riding Local Research Ethics Committee for this study (LEC/05/02/091, 06/09/2003). Tumour patients were already on steroid treatment (either oral or intravenous Dexamethasone $4 \mathrm{mg}$ x 4/day) for $48 \mathrm{~h}$ prior to venepuncture. Patients were divided on the basis of histopathological reports made according to the WHO Brain Tumour Classification (19). A summary of the patients' details and clinicopathological features are given in Table I.

Serum samples obtained via venepuncture into a 7-ml serum separation tube were immediately spun in a centrifuge at $150 \mathrm{xg}$ for $10 \mathrm{~min}$ to separate serum which was then aliquoted and frozen in $1.5-\mathrm{ml}$ polypropylene tubes (Sardstedt tubes) at $-80^{\circ} \mathrm{C}$.

IL12 and IL10 determination. Quantitative sandwich ELISA assays (BioSource) were used to analyse IL12 (IL12 UltraSensitive ELISA cat no. KHC0124) which measures both IL12p70 and p40) and IL10 (IL10 UltraSensitive ELISA cat no. KHC0104). The lower level of detection for IL12 and IL10 was 1.56 and $0.78 \mathrm{pg} / \mathrm{ml}$, respectively. All samples were analysed in duplicate and freeze-thawing of samples was avoided.

Proteoplex $^{\mathrm{TM}}$ analysis. The Proteoplex ${ }^{\mathrm{TM}}$ 16-well human cytokine array kit (Merck Biosciences) was used according to manufacturer's protocol to simultaneously measure the following 12 cytokines: IL-1 $\alpha$, IL-1ß, IL-2, IL-4, IL-6, IL-7, IL-8, IL-10, IL-12p70, GM-CSF, IFN- $\gamma$ and TNF- $\alpha$ in a single

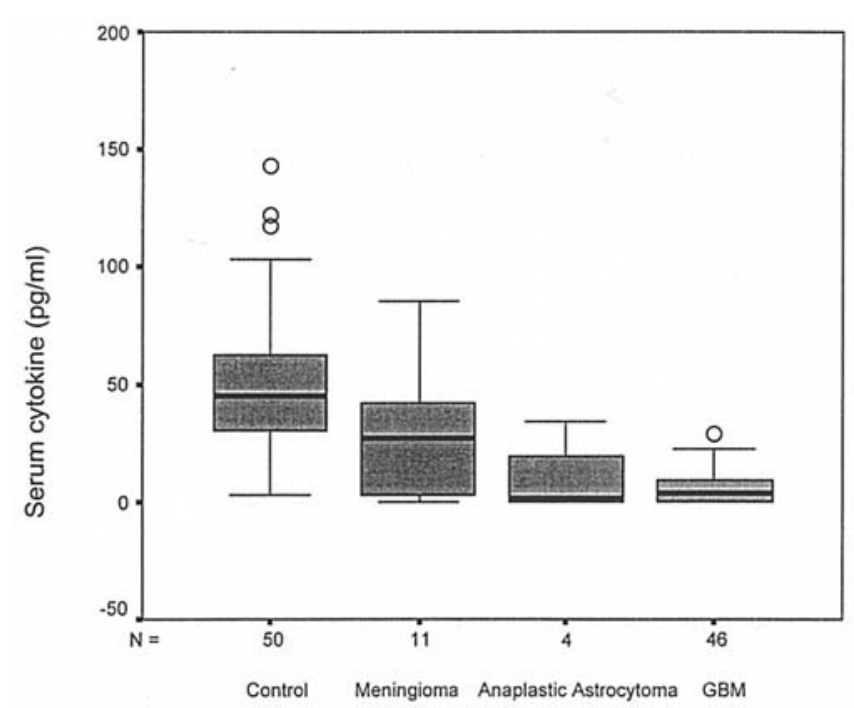

Figure 1. Box plots comparing serum IL-12 in patients with meningioma, anaplastic astrocytoma, GBM and controls. Median levels of serum IL-12 = $45.3 \mathrm{pg} / \mathrm{ml}$ (control), $27.4 \mathrm{pg} / \mathrm{ml}$ (meningioma), $1.94 \mathrm{pg} / \mathrm{ml}$ (anaplastic astrocytoma) and $4.03 \mathrm{pg} / \mathrm{ml}(\mathrm{GBM})$. Significant decreases were seen in all groups compared with the control group; meningioma $(\mathrm{p}=0.03)$, anaplastic astrocytoma $(\mathrm{p}<0.001)$, GBM $(\mathrm{p}<0.001)$; Mann-Whitney U test.

serum sample (50 $\mu 1)$. The lower level of detection was $5 \mathrm{pg} /$ $\mathrm{ml}$. Serum samples from seven individual patients, taken pre-, 24 and $48 \mathrm{~h}$ post commencement of dexamethasone (either oral or intravenous $4 \mathrm{mg} \mathrm{x} 4$ times daily) were analysed.

Statistical analysis. Median serum cytokine levels were compared between the tumour patient and control group using a non-parametric independent T-test (Mann-Whitney U) with significance being determined as $p \leq 0.05$. Cytokine differences pre- and post-steroid administration were compared using the Wilcoxon signed rank test. Statistical advice was provided by Dr E. Gardiner (Biomedical statistician).

\section{Results}

All patients and controls had detectable levels of IL-12; however, all groups of patients had significantly lower levels when compared with controls, with the most advanced tumours having the lowest levels (Fig. 1). Conversely, the trend in serum IL-10 levels was for raised levels being associated with tumour, with levels in the anaplastic astrocytoma and GBM group both being significantly higher than non-tumour controls (Fig. 2). One further interesting observation is that 


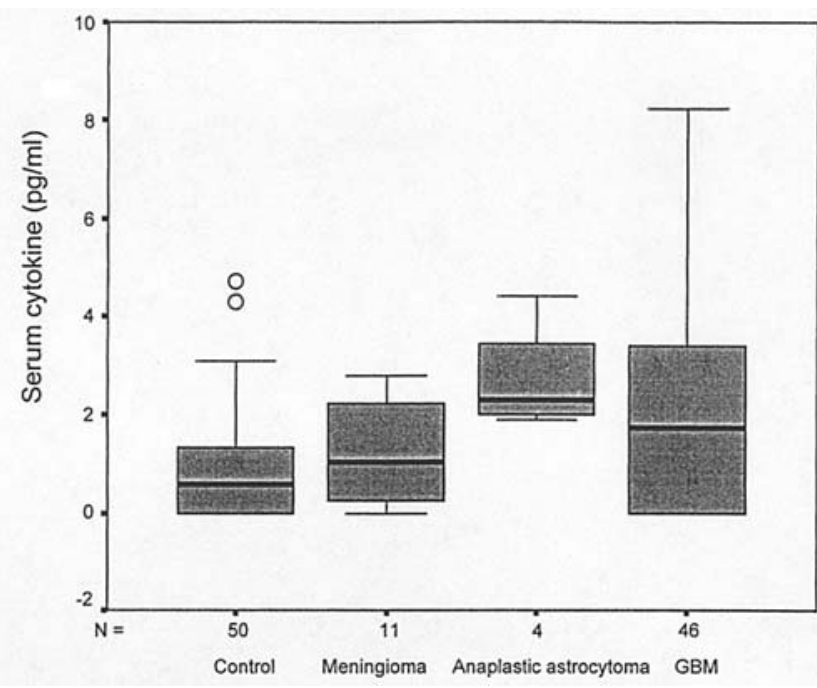

Figure 2. Box plots comparing serum IL-10 in patients with meningioma, anaplastic astrocytoma, GBM and controls. Median levels of serum IL-10 = $0.58 \mathrm{pg} / \mathrm{ml}$ (control), $1.04 \mathrm{pg} / \mathrm{ml}$ (meningioma), $2.3 \mathrm{pg} / \mathrm{ml}$ (anaplastic astrocytoma) and $1.74 \mathrm{pg} / \mathrm{ml}(\mathrm{GBM})$. Significant increase was seen in the anaplastic astrocytoma $(\mathrm{p}=0.02)$ and GBM $(\mathrm{p}=0.03)$ group; Mann-Whitney U test.

$25 \%$ of the tumour group and $38 \%(19 / 50)$ of the control group measured below the level of detection $(<0.78 \mathrm{pg} / \mathrm{ml})$ of the ELISA kit. In summary, patients with anaplastic astrocytoma or GBM showed both a significant decrease in serum IL-12 levels (both $\mathrm{p}<0.001)$ and significant increase in serum IL-10 ( $\mathrm{p}=0.02, \mathrm{p}=0.03$, respectively).

Analysis of changes in serum cytokines pre- and poststeroid treatment by Proteoplex assay showed no statistically significant changes in any cytokine up to $48 \mathrm{~h}$ after commencement of dexamethasome (data not shown).

\section{Discussion}

We have clearly demonstrated, in this cohort of primary brain tumours, that there is an imbalance in the systemic IL-12/ IL-10 balance in comparison with non-tumour controls, which is highly likely to effect immune-regulation. We have also shown that the differences in cytokine production are not affected in the first $48 \mathrm{~h}$ by steroid administration.

Although, to our knowledge, this is the first report of its type investigating the balance of circulating IL-12 and IL-10 levels in brain tumour patients, there has been one previous study on circulating IL-12 in patients with brain tumours. Salmaggi et al performed a small-scale study designed to compare levels of a group of pro-inflammatory and potent angiogenic cytokines in serum, plasma and cyst tumour fluid in brain tumour patients with a control group; IL-12 was included (20). Their study cohort comprised recurrent anaplastic astrocytoma $(n=7)$, GBM $(n=12)$ and recurrent glioblastoma multiforme $(n=26)$. A significant reduction in serum IL-12 compared with age-matched controls (mean $=45.6 \pm$ SEM 7.76 vs. $79.78 \pm 6.32 \mathrm{pg} / \mathrm{ml}$ ) was seen in agreement with the findings of the present study. No previous studies have analysed the Th1/Th2 balance in plasma or serum of meningioma patients.

A number of similar studies on this crucial cytokine balance have been reported in other systemic tumours. Sparano et al
(21) studied the plasma concentrations of IL-4, -6, -10 and -12 in 58 patients with squamous cell carcinoma of the head and neck. For IL-12, an OPT-EIA, BD PharMingen kit was used which only detected IL-12p40, rather than an ELISA which measures both free IL-12p40 and heterodimeric IL-12p70. The concentrations of IL- 12 were greater in patients without nodal metastases and with $\mathrm{T}_{1} / \mathrm{T}_{2}$ tumours. The concentration of IL-10 was lower in these patients. However, IL-10 levels were greater in patients with nodal metastasis with $T_{3} / T_{4}$ tumours and, conversely, the concentration of IL-12 was lower in these patients. Hence, patients with advanced HNSCC apparently have a diminished Th1 immune response and a stronger Th2 immune response when compared to that of patients with less advanced disease. Similarly, Lathers et al (22) showed incomplete Th2 skewing of cytokines in plasma of 101 patients with squamous cell carcinoma of the head and neck. In this study, plasma levels of three Th1 cytokines (IFN- $\gamma$, IL- 2 and GM-CSF) and three Th2 cytokines (IL-4, -6 and -10 ) were studied (22). Here levels of IL-4, -6 and -10 were significantly increased. However, the Th1 cytokine picture was more complicated as IL-2 and GM-CSF were also significantly raised, although IFN- $\gamma$ was significantly decreased. Lathers et al (22) concluded that these results suggested a skewing towards Th2, with the Th1 shift being incomplete. Finally, a previous study in Hull has shown that patients with large $\left(\mathrm{T}_{3}\right.$ or $\left.\mathrm{T}_{4}\right)$ or advanced (Dukes' $\mathrm{C}$ ) colorectal tumours have decreased levels of IL-12 production by Staphylococcus aureus Cowans Strain 1 (SAC) stimulated PBMC and raised serum IL-10 levels in comparison with normal controls (3). The above studies, demonstrate that an apparent imbalance is widely observed in various tumours, most usually with advanced disease showing the more prominent aberrations.

It must be noted, however, that an apparently contradictory study has also been reported. Tsuboi et al (18) showed that serum IL-12 and IL-18 levels were significantly higher in 70 patients with oesophageal carcinoma compared with 15 healthy controls and that there was a positive correlation between serum IL-12 and IL-18 levels, which correlated with tumour growth and progression. Surprisingly, although serum IL-12 and IL-18 levels increased, tumour activity did not appear to be suppressed. Tsuboi and his colleagues postulated that the anti-tumour effect of IL-12 only occurs at an early stage rather than at a later time-point. With disease progression, tumour volume might overcome the immune response and, therefore, the clinical stage may progress in spite of an apparent increase in serum Th1 levels. It is unfortunate that the levels of serum IL10 were not measured concomitantly and further work is required to see if this effect is specific to oesophageal cancer.

The studies discussed above, like the one reported here, have analysed either serum or plasma Th1/Th2 cytokines. Two other studies have analysed the functional effects on peripheral blood mononuclear cells (PBMC) in order to determine whether there is an increased or impaired cytokine production. The first of these has investigated the influence(s) of glioblastoma on the immune response by studying the effects of tumour-derived factors on cytokine production by PBMC. Zou et al isolated PBMC from healthy donors and incubated these cells with human glioblastoma culture supernatant (23). 
This resulted in an increased production of IL-10 and IL-6 and decreased production of IL-12, IFN- $\gamma$ and TNF- $\alpha$. The group concluded that monocytes can serve as an intermediate in determining the nature of the immune response by receiving signals from the tumour and other components of the host immune system. This study also suggests that the systemic changes in cytokines observed in cancer patients may not solely be due to tumour production, but may also be due to 'reprogramming' of key populations of immune cells. In a similar study, Shibata et al (17) also examined the production of cytokines (IL-4, -6, -10, -12 and IFN- $\gamma$ ) by stimulating PBMC, using cells isolated from cancer patients (colorectal $n=30$, gastric $n=25)$ or normal controls $(n=39)$ stimulated with the non-specific activators, phytohemagglutinin or S.aureus. Interestingly, the production of IL-12 decreased significantly with advancing disease and was lowest in the patients with distant metastases and cachexia. Initially, no differences were observed in the levels of either of the Th2 cytokines (IL-4 and IL-10) between patient groups; however, production of these cytokines had increased when the same cachectic patients were retested after 2.5 months. The authors' findings indicate that there is a move away from a Th1 profile at a relatively early stage of disease, which would agree with the findings of Tsuboi et al (18), whereas a swing to a Th2 profile only seems to increase in the more advanced stage of the disease, i.e. a consequence of tumour progression rather than a cause. Whether such changes occur in brain tumours that lack lymphatic drainage remains to be elucidated.

The study here suggests that there is a Th1/Th2 cytokine imbalance in patients with brain tumours which is similar to that observed in studies on several other tumour types. A Th2 type cytokine shift, which would support humoral immunity at the expense of cell-mediated immune response, is observed in patients with advanced disease and may well contribute to the inefficient anti-tumour response generated in these patients. Further studies of the complex interaction of cytokines and their receptors between neoplastic and immune cells may provide useful information toward the search for effective immunotherapies. Future work will involve assessment of post-operative status of these cytokines in patients with brain tumours as previous work on renal cell carcinoma and melanoma has shown that removal of the tumour from patients with active disease results in the Th1/Th2 balance becoming less polarised towards Th2 (24). In addition, it will prove highly enlightening to investigate further PBMC function in an attempt to clarify what effect the changes in cytokine milieu are having on the immune system of patients with intracranial tumours.

\section{References}

1. www.statistics.gov.uk.

2. Parney IF, Hao C and Pertuk KC: Glioma immunology and immunotherapy. Neurosurgery 46: 778-798, 2000.

3. O'Hara RJ, Greenman J, McDonald AW, Gaskell KM, Topping KP, Duthie GS, Kerin MJ, Lee PW and Monson JRT: Advanced colorectal cancer is associated with impaired interleukin-12 and enhanced interleukin-10 production. Clin Cancer Res 4: 1943-1948, 1998.

4. Trinchieri G: Interleukin-12: a proinflammatory cytokine with immunoregulatory functions that bridge innate resistance and antigen-specific adaptive immunity. Annu Rev Immunol 13: 251-276, 1995.
5. Watford WT, Moriguchi M, Morinobu A and O'Shea JJ: The biology of IL-12: coordinating innate and adaptive immune responses. Cytokine Growth Factor Rev 14: 361-368, 2003.

6. Fukao T, Frucht DM, Yap G, Gadina M, O'Shea JJ and Koyasu S: Inducible expression of Stat 4 in dendritic cells and macrophages and its critical role in innate and adaptive immune responses. J Immunol 166: 4446-4455, 2001.

7. Frucht DM, Fukao T, Bogdan C, Schindler H, O'Shea JJ and Koyasu S: IFN-gamma production by antigen-presenting cells: mechanisms emerge. Trends Immunol 22: 556-560, 2001.

8. O'Shea JJ and Paul WE: Regulation of T(H)1 differentiation controlling the controllers. Nat Immunol 3: 506-508, 2002.

9. Nishimura T, Watanabe K, Lee U, Yahata T, Ando K, Kimura M, Hiroyama Y, Kobayashi M, Herrmann SH and Habu S: Systemic in vivo antitumour activity of interleukin-12 against both transplantable and primary tumour. Immunol Lett 48: 149-152, 1995.

10. Noguchi Y, Richards EC, Chen YT and Old LJ: Influence of interleukin 12 on p53 peptide vaccination against established Meth A sarcoma. Proc Natl Acad Sci USA 92: 2219-2223, 1995.

11. Boggio K, Nicoletti G, Di Carlo E, Cavallo F, Landuzzi L, Melani C, Giovarelli M, Rossi I, Nanni P, De Giovanni C, Bouchard P, Wolf S, Modesti A, Musiani P, Lollini PL, Colombo MP and Forni G: Interleukin 12-mediated prevention of spontaneous mammary adenocarcinomas in two lines of Her-2/ neu transgenic mice. J Exp Med 188: 589-596, 1998.

12. Cavallo F, Signorelli P, Giovarelli M, Musiani P, Modesti A, Brunda MJ, Colombo MP and Forni G: Antitumor efficacy of adenocarcinoma cells engineered to produce interleukin 12 (IL-12) or other cytokines compared with exogenous IL-12. J Natl Cancer Inst 89: 1049-1058, 1997.

13. Rakhmilevich AL, Janssen K, Turner J, Culp J and Yang NS: Cytokine gene therapy of cancer using gene gun technology: superior antitumour activity of interleukin-12. Hum Gene Ther 8: 1303-1311, 1997.

14. Fiorentino DF, Bond MW and Mosmann TR: Two types of mouse T helper cell: IV. Th2 clones secrete a factor that inhibits cytokine production by Th1 clones. J Exp Med 170: 2081-2095, 1989.

15. Nitta T, Hishii M, Sato K and Okumura K: Selective expression of IL-10 gene within GBM. Brain Res 649: 122-128, 1994.

16. Dummer W, Becker JC, Schwaaf A, Leverkus M, Moll T and Brocker EB: Elevated serum levels of interleukin-10 in patients with metastatic malignant melanoma. Melanoma Res 5: 67-68, 1995.

17. Shibata M, Nezu T, Kanou H, Abe H, Takekawa M and Fukuzawa M: Decreased production of interleukin-12 and type 2 immune responses are marked in cachectic patients with colorectal and gastric cancer. J Clin Gastroenterol 34: 416-420, 2002.

18. Tsuboi K, Miyazaki T, Nakajima M, Fukai Y, Masuda N, Manda R, Fukuchi M, Kato H and Kuwano H: Serum interleukin-12 and interleukin-18 levels as a tumour marker in patients with esophageal carcinoma. Cancer Letts 205: 207-214, 2004.

19. Kleiheus P and Cavenee WK: World Health Organization classification of tumours of the nervous system. IRAC/WHO, Lyon 2000.

20. Salmaggi A, Eoli M, Frigerio S, Silvani A, Gelati M, Corsini E, Broggi $G$ and Boiardi A: Intracavitary VEGF, $3 F G F$, IL-8, IL-12 levels in primary and recurrent malignant glioma. J Neurooncol 62: 297-303, 2003.

21. Sparano A, Lathers DM, Achille N, Petruzzelli GJ and Young MR: Modulation of Th1 and Th2 cytokine profiles and their association with advanced head and neck squamous cell carcinoma. Otolaryngol Head Neck Surg 131: 573-576, 2004.

22. Lathers DM, Achille NJ and Young MR: Incomplete Th2 skewing of cytokines in plasma of patients with squamous cell carcinoma of the head and neck. Hum Immunol 64: 1160-1166, 2003.

23. Zou JP, Morford LA, Chougnet C, Dix AR, Brooks AG, Torres N, Shurman JD, Coligan JE, Brooks WH, Roszman TL and Shearer GM: Human glioma-induced immunosuppression involves soluble factor(s) that alters monocyte cytokine profile and surface markers. J Immunol 162: 4882-4892, 1999.

24. Tatsumi T, Kierstead LS, Ranieri E, Gesualdo L, Schena FP, Finke JH, Bukowski RM, Mueller-Berghaus J, Kirkwood JM, Kwok WW and Storkus WJ: Disease-associated bias in T helper type (Th1)/Th2 CD4(+) T cell responses against MAGE-6 in HLA-DRB $1 * 0401(+)$ patients with renal cell carcinoma or melanoma. J Exp Med 196: 619-628, 2002. 